\title{
Signal Change in the Mammillary Bodies after Perinatal Asphyxia
}

\author{
(D) M. Molavi, (D)S.D. Vann, (D)L.S. de Vries, (DF. Groenendaal, and (D) M. Lequin
}

\begin{abstract}
BACKGROUND AND PURPOSE: Research into memory deficits associated with hypoxic-ischemic encephalopathy has typically focused on the hippocampus, but there is emerging evidence that the medial diencephalon may also be compromised. We hypothesized that mammillary body damage occurs in perinatal asphyxia, potentially resulting in mammillary body atrophy and subsequent memory impairment.
\end{abstract}

MATERIALS AND METHODS: We retrospectively reviewed brain MRIs of 235 clinically confirmed full-term patients with hypoxic-ischemic encephalopathy acquired at a single center during 2004-2017. MRIs were performed within 10 days of birth (median, 6; interquartile range, 2). Two radiologists independently assessed the mammillary bodies for abnormal signal on T2-weighted and DWI sequences. Follow-up MRIs were available for 9 patients; these were examined for evidence of mammillary body and hippocampal atrophy.

RESULTS: In 31 neonates (13.2\%), abnormal high mammillary body signal was seen on T2-weighted sequences, 4 with mild, 25 with moderate, and 2 with severe hypoxic-ischemic encephalopathy. In addition, restricted diffusion was seen in 6 neonates who had MR imaging between days 5 and 7. For these 31 neonates, the most common MR imaging pattern (41.9\%) was abnormal signal restricted to the mammillary bodies with the rest of the brain appearing normal. Follow-up MRIs were available for 9 patients: 8 acquired between 3 and 19 months and 1 acquired at 7.5 years. There was mammillary body atrophy in 8 of the 9 follow-up MRIs.

CONCLUSIONS: Approximately $13 \%$ of full-term infants with hypoxic-ischemic encephalopathy showed abnormal high mammillary body signal on T2-weighted images during the acute phase, which progressed to mammillary body atrophy in all but 1 of the infants who had follow-up MR imaging. This mammillary body involvement does not appear to be related to the severity of encephalopathy, MR imaging patterns of hypoxic-ischemic encephalopathy, or pathology elsewhere in the brain.

ABBREVIATIONS: $\mathrm{MB}=$ mammillary body; $\mathrm{HIE}=$ hypoxic-ischemic encephalopathy; $\mathrm{IQ}=$ intelligent quotient

$\mathbf{T}$ he mammillary bodies (MBs) are a pair of small projections situated at the posterior margin of the hypothalamus at the base of the brain, named "mammillary" because of their shape. They have repeatedly been implicated in mnemonic processes and are particularly important for episodic (ie, event) memory. ${ }^{1-3}$

Received April 17, 2019; accepted after revision August 8.

From the Departments of Radiology and Neonatology (M.M., L.S.d.V., F.G., M.L.) Wilhelmina Children's Hospital, University Medical Center Utrecht and Utrecht University, Utrecht, the Netherlands; and School of Psychology (S.D.V.), Cardiff University, Cardiff, UK.

S.D. Vann is funded by a Wellcome Trust Senior Research Fellowship in Biomedical Sciences (WT 12273/Z/18/Z).

Please address correspondence to Maarten Lequin, MD, Department of Radiology, Wilhelmina Children's Hospital, University Medical Center Utrecht and Utrecht University, Lundlaan 6, 3584 EA Utrecht, the Netherlands; e-mail: M.H.Lequin@umcutrecht.nl

--3 Indicates open access to non-subscribers at www.ajnr.org

Indicates article with supplemental on-line table.

http://dx.doi.org/10.3174/ajnr.A6232
MB damage is typically associated with conditions linked to thiamine deficiency, in particular Korsakoff syndrome; ${ }^{4}$ however, it appears that the MBs may be implicated in a greater number of neurologic conditions than previously appreciated. ${ }^{5-7}$

In infants, brain areas typically vulnerable to hypoxic-ischemic encephalopathy (HIE) include the deep gray nuclei, cortex, and white matter, depending on the severity and duration of the insult. ${ }^{8}$ In addition, the limbic system is involved in some patients, showing diffusion restriction on DWI, particularly in the hippocampal region. ${ }^{9,10}$ Full-term neonates with perinatal asphyxia can exhibit hippocampal volume loss from childhood onward as a consequence of possible cytotoxic injury at the time of hypoxia-ischemia. ${ }^{11}$ Developmental amnesia, a disorder primarily affecting episodic memory, typically occurs as a result of early-onset hypoxia-induced damage to the hippocampus. ${ }^{12}$ However, the extent of hippocampal pathology does not always predict the severity of subsequent memory impairment. ${ }^{13}$ Consistent with this finding, a recent study by Dzieciol et $\mathrm{al}^{14}$ 

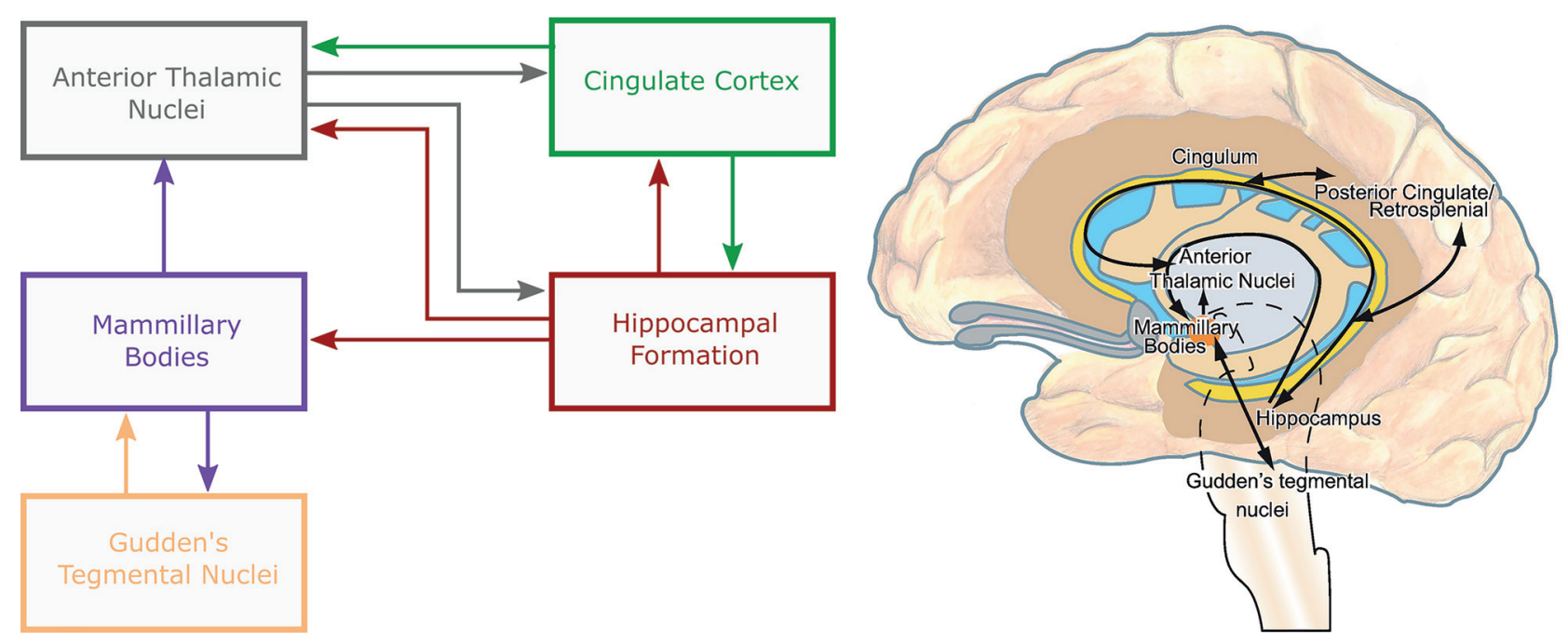

FIG 1. Schematic of the extended Papez system highlighting the principal connections of the mammillary bodies.

examined MR images of 18 patients with developmental amnesia (11-35 years of age) and uncovered a marked degree of atrophy in the MBs in two-thirds of the patients. However, because the MR images were acquired a number of years following the hypoxic-ischemic event, the authors were unable to conclude whether the $\mathrm{MB}$ atrophy was directly related to the hypoxic-ischemic episode or due to subsequent degeneration. ${ }^{14,15}$

The aim of the present study, therefore, was to examine whether the MBs are affected in infants with HIE. Given previous reports of $\mathrm{MB}$ sensitivity to hypoxia in adults, ${ }^{16,17}$ we hypothesized that the MBs would be directly compromised in HIE in neonates with changes evident on MRIs acquired during the neonatal period. We assessed MR imaging signal change in the MBs of infants with perinatal asphyxia. The sample group included infants treated with therapeutic hypothermia and those who did not undergo hypothermia treatment. In infants with abnormal MB signal, the wider Papez circuit (eg, hippocampal formation, fornix, cingulate gyri, and the anterior thalamic nuclei; Fig 1) was also examined for abnormalities. When possible, follow-up MR images were evaluated to determine whether abnormal MB signal during the acute phase was associated with subsequent $\mathrm{MB}$ atrophy.

\section{MATERIALS AND METHODS \\ Patients}

In this retrospective, observational, single-center study, a total of 235 full-term neonates were enrolled, meeting the previously described clinical criteria of $\mathrm{HIE}^{18}$ and having undergone brain MR imaging at the University Medical Center Utrecht, Wilhelmina Children's Hospital, during 2004-2017. The patients have been included in previous studies. ${ }^{8,9,19-22}$ A waiver of informed consent was obtained, according to European regulations, because the present study involved the analysis of anonymized data.

The total group included 2 subsets. The first subset comprised 70 neonates, born between 2004 and January 2008, who did not receive therapeutic hypothermia. The second subset comprised 165 neonates, born between February 2008 and
2017, who were treated with whole-body hypothermia. Clinical data were retrieved from the patients' records.

\section{MR Imaging Acquisition and Assessment}

Cerebral MR imaging was performed within 3-10 days of birth (median, 6; interquartile range, 2), on days 3-9 (infants with normothermia), and days 5-10 (infants with hypothermia). ${ }^{20,23} \mathrm{MR}$ imaging protocols have been described previously. ${ }^{19}$ Briefly, MR imaging was performed on a $1.5 \mathrm{~T}$ or $3 \mathrm{~T}$ MR imaging system (Achieva Philips Healthcare Best, the Netherlands). The scanning protocol included sagittal T1 before 2013 and sagittal T2weighted imaging after 2013 (sagittal T2-weighted images were available for 64 patients). This was followed by axial T1-weighted and T2-weighted sequences with $2-\mathrm{mm}$ section thickness. In addition, an echo-planar imaging technique was used for diffusion-weighted imaging, with axial 4-mm section thickness, $0-\mathrm{mm}$ section gap, and b-values of 0 and $1000 \mathrm{~s} / \mathrm{mm}^{2}$ (1.5T) or b-values of 0 and $800 \mathrm{~s} / \mathrm{mm}^{2}(3 \mathrm{~T})$.

All MRIs were retrospectively screened, independently, by 2 radiologists (M.L. and M.M., with experience in reporting neonatal MRIs for 20 years and 3 years, respectively). The MBs were assessed for abnormal high signal on the T2-weighted images and/or abnormal signal on DWI (high signal on the trace maps and low signal on the ADC maps). Given the size and location of the MBs, it was important to reduce the likelihood of identifying artifactual signal due to partial volume effects. Therefore, an increased signal intensity had to be clearly visible over 2 consecutive T2-weighted slices. This increased signal intensity was then confirmed by subjectively assessing swelling in MBs and/or observing decreased signal intensity on T1-weighted images. All available sections and directions were evaluated. DWI was considered to have positive findings when signal changes (high signal on trace maps and low signal on ADC maps) were present over 2 consecutive slices. The section thickness in DWI sequences was $4 \mathrm{~mm}$; therefore, they were more prone to artifactual signals. Thus, only infants with obvious signal changes on T2weighted images were included (Fig 2). In addition to examining the $\mathrm{MBs}$, images were examined for abnormal signal 

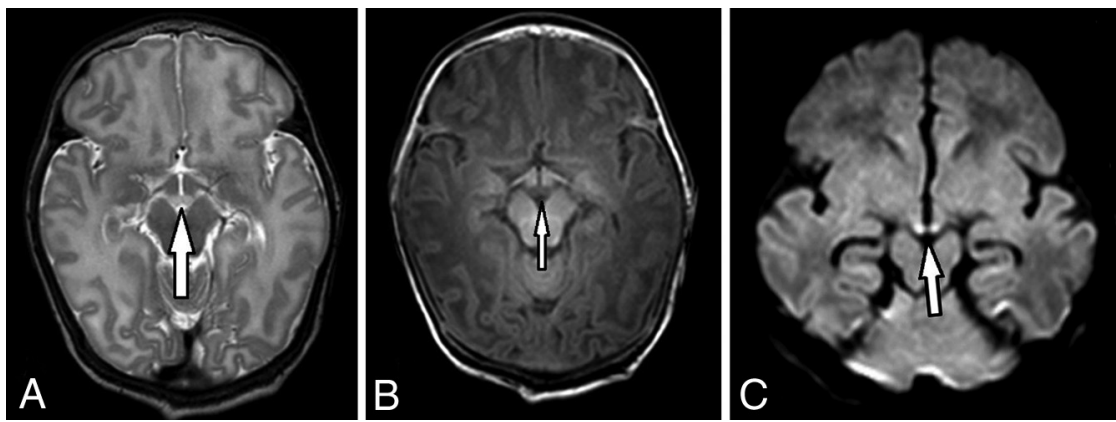

FIG 2. Arrows indicate the following: abnormally high-signal MBs on an axial T2-weighted image $(A)$; abnormally low-signal MBs on an axial Tl-weighted image (B); and abnormally high-signal MBs on DWI, b-value $=1000$, accompanied by low ADC (not shown) (C).
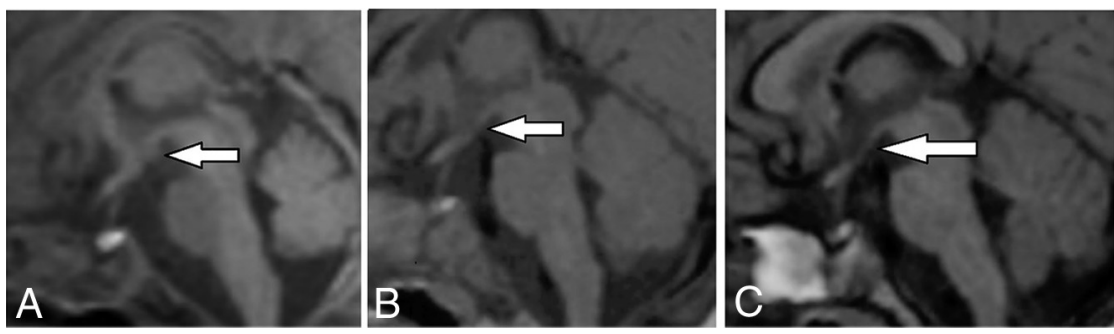

FIG 3. Sagittal T1-weighted MR imaging. The arrows indicate abnormal, low signal in MBs at 6 days $(A)$ and $M B$ atrophy at $3(B)$ and $19(C)$ months.

Table 1: General demographic and clinical findings of neonates with high MB signal on T2-weighted images

\begin{tabular}{lc}
\hline \multicolumn{2}{c}{ Cases with T2-Weighted High-Signal MB ( $\boldsymbol{n}=31)$} \\
\hline Sex, male/female & $15: 16$ \\
Gestational age (mean) (wk) & $40 \pm 2.0$ \\
Birth weight (mean) (kg) & $3113 \pm 556$ \\
Apgar 1 min (median) (IQR) & $2(3)$ \\
Apgar 5 min (median) (IQR) & $5(4)$ \\
Age at first MR imaging in days after birth (median) & $6(2)$ \\
$\quad$ (IQR) & $17 / 14$ \\
Field strength of MR imaging (1.5T/3T) & $4 / 25 / 2$ \\
Grade of encephalopathy (Sarnat I/II/III) & $18(58)$ \\
Hypothermia (\%) & $4(13)$ \\
Died (\%) & $21(68)$ \\
Normal findings on clinical follow-up (\%) & $5(16)$ \\
Abnormal findings on clinical follow-up (\%) & $8(26)$ \\
Severe hyponatremia (<130 mEq/L) (\%) & $3(10)$ \\
Systemic infection (\%) &
\end{tabular}

Note:-IQR indicates interquartile range.

intensity in other constituents of the extended Papez circuit (Fig $1):{ }^{24,25}$ hippocampi, fornices, anterior nuclei of the thalamus, and cingulate gyri. The tegmental nuclei in the brain stem were too small to assess due to a section thickness of $2 \mathrm{~mm}$ (T2) or more (DWI).

Nine patients underwent follow-up MR imaging: 8 at 3-19 months and 1 at 7.5 years. Two radiologists (M.L. and M.M.) independently assessed the scans for evidence of subjectively judged $\mathrm{MB}$ atrophy. The MBs were considered atrophic if there was a lack of protrusion clearly seen on sagittal sections (Fig 3). The hippocampi were also examined for signs of atrophy.

\section{Neurodevelopmental Follow-Up}

Clinical follow-up data were reviewed, when available, which focused on intelligence quotient (IQ) and motor and neurologic functions. The assessments provide a general evaluation of development but do not specifically measure memory. The tests administered were the following: Bayley Scales of Infant Development, Griffiths Mental Development Scales, Wechsler Preschool and Primary Scales of Intelligence, and Movement Assessment Battery for Children. Patients were tested with the Bayley Scales of Infant Development and Griffiths Mental Development Scales at 24 months and the Wechsler Preschool and Primary Scales of Intelligence and Movement Assessment Battery for Children test at 5 years.

Descriptive statistics were calculated using SPSS, Version 21 (IBM, Armonk, New York).

\section{RESULTS}

An increased T2-weighted signal was noted in the MBs in 31 of the 235 infants (13.2\%) (Fig 2). Details of these patients are presented in Table 1. T2-weighted hyperintensity was observed in the MBs of 18 infants (10.9\%) with therapeutic hypothermia and 13 infants (18.6\%) without therapeutic hypothermia. Concomitant diffusion restriction was found in 6 of these infants (4 with therapeutic hypothermia and 2 without). The day of the MR imaging acquisition is shown in Fig 4; abnormal MB signal could be identified across imaging days 3-10.

In $13(41.9 \%)$ of these 31 patients, the MR imaging findings appeared otherwise normal. The occurrence rates of common pathology patterns associated with hypoxia are summarized in Table 2. When additional damage was present, the watershed predominant pattern of damage occurred most commonly (in $29 \%$ of infants). Involvement of other structures within the Papez circuit is shown in Table 3; hippocampal involvement was the most common presentation, occurring in approximately onethird of infants.

Of the 31 patients, 8 patients had at least 1 follow-up MR scan ranging between 3 and 19 months after the first MR scan and 1 patient had follow-up MR imaging at 7.5 years. Clinical findings for patients with available follow-up MRIs are provided in Table 4. MB atrophy was observed in 8 of 9 infants with follow-up MR imaging (the On-line Table); hippocampal atrophy was present in 6 of these patients.

\section{Clinical Follow-Up Results}

Of the 31 identified neonates with abnormal MB signal, 4 died before discharge due to redirection of care because of severe brain injury or associated multiorgan failure. Most $(n=21)$ were assessed as "healthy" at early follow-up. Five patients had an 


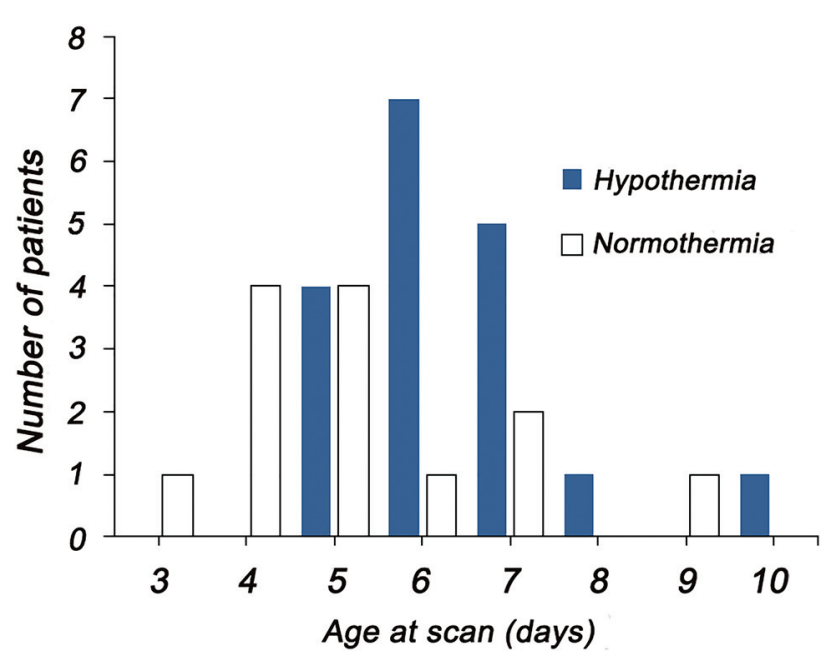

FIG 4. Day (postbirth) of initial MR imaging in patients with hypothermia treatment or without (normothermia). The day of scanning was typically days 5-7 for the hypothermia-treated patients, whereas there was a greater range of scanning days for patients with normothermia.

Table 2: MR imaging pattern of hypoxic-ischemic lesions

\begin{tabular}{lc}
\hline \multicolumn{1}{c}{ Pattern } \\
\hline NL & $13(41.9 \%)$ \\
BGT & $5(16.1 \%)$ \\
WS & $9(29 \%)$ \\
NT & $3(9.7 \%)$ \\
Large subdural hematoma & $1(3.2 \%)$ \\
Total & 31 \\
\hline
\end{tabular}

Note:-NL indicates normal; BGT, basal ganglia and thalamus; WS, watershed; NT, near total.

Table 3: Involvement of additional structures within the Papez circuit

\begin{tabular}{lc}
\hline Additional Structures & \\
\hline F & 1 \\
HC & 4 \\
AT & 0 \\
CG & 1 \\
AT/F & 1 \\
HC/F & 7 \\
More than 2 involved & 4 \\
None & $13(41.9 \%)$ \\
Total & 31 \\
\hline
\end{tabular}

Note:-F indicates fornix; HC, hippocampus; AT, anterior thalamus; CG, cingulate gyrus.

abnormal outcome. Three of these patients had a total IQ on the Wechsler Preschool and Primary Scales of Intelligence of $<85$ at 5 years. In one of these patients, this low IQ was associated with hearing loss and concentration problems. The fourth patient had cerebral visual impairment, though normal performance on IQ tests. One patient showed some behavioral problems but was otherwise healthy. The final patient was younger than 12 months, too young to be assessed.

\section{DISCUSSION}

Improvements in medical care have resulted in an increasing number of children surviving perinatal asphyxia. Therefore, there is a growing need to identify the sites of neuropathology following hypoxic-ischemic episodes in neonates and how this may contribute to subsequent cognitive impairments.

The present study focused on the MBs because atrophy in this region has been reported in an older group of patients who had a hypoxic-ischemic episode in childhood. ${ }^{14}$ However, the suggestion from this earlier study was that MB atrophy was a result of degeneration following primary damage to the hippocampus or limbic cortex. ${ }^{14}$ Contrary to this explanation, we found evidence of MR imaging signal change in the MBs of neonates with HIE with a T2 signal rise in $13 \%$ of patients with HIE. In 13/31 infants, the signal intensity abnormalities were seen only in the MBs, making them almost certain to be the site of primary injury. It is, therefore, possible to conclude that the MBs can be directly affected in HIE. Where signal change is observed in multiple regions, it is possible that the $\mathrm{MB}$ involvement is secondary. However, if this were the case, on the basis of known connectivity, the most likely site of primary pathology would be the hippocampal formation. ${ }^{1}$ A study in rhesus monkeys found no abnormal MB signal 6 days following experimental hippocampal lesions, ${ }^{26}$ again suggesting that in the timescale of the current study, the $\mathrm{MB}$ involvement is likely to be a primary effect. Together, the implication is that while secondary degeneration may cause additional pathology within the MBs at longer time periods, this structure is nonetheless sensitive to direct effects of hypoxic-ischemic episodes in neonates.

To the best of our knowledge, this is the first study to assess MB MR imaging signal changes in neonates with HIE. Therefore, the current findings add to the increasing body of literature from adult patients in whom there is antemortem and postmortem evidence of MB necrosis following severe HIE. ${ }^{17,27}$ Reduced MB volume has also been reported in patients with obstructive sleep apnea ${ }^{6}$ and those with heart failure, ${ }^{28}$ again conditions linked to both acute and chronic hypoxia. The findings from both neonatal and adult patients further highlight the need to assess MB status in conditions with both acute and chronic low brain oxygen levels.

MB pathology is most commonly associated with, or exaggerated by, thiamine deficiency. ${ }^{4,29-31}$ Thiamine is essential for intracellular metabolism and acts as a neuroprotective agent against oxidative stress. Reduced thiamine may exacerbate the effects of hypoxia on MBs and has been indicated as a possible contributing factor in a number of studies in which adult MB atrophy has been found following hypoxic-ischemic episodes. ${ }^{6,17,27,28}$ However, thiamine deficiency is not a prerequisite for hypoxiarelated $\mathrm{MB}$ atrophy in adults. ${ }^{16}$ Furthermore, all patients in the present study were administered thiamine following birth; therefore, thiamine deficiency is unlikely to be a contributing factor to the increased signal intensity observed in the current cohort.

We also checked for signs of cerebritis, which may be a possible cause of $\mathrm{MB}$ signal rise and DWI restriction. No evidence of cerebral infection was found either on MR images or clinically, except in 3 patients with signs of a systemic infection. None of the 31 patients underwent a lumbar puncture, so a CNS infection cannot be ruled out completely. However, it seems unlikely, looking at the clinical outcome and, in some patients, follow-up MR imaging findings. 
Surprisingly, we noticed $\mathrm{MB}$ involvement not only in patients with severe HIE but also in those with milder HIE. On the other hand, it was of interest that some infants with severe HIE did not show any MB abnormality on T2 or DWI series. These preliminary findings would suggest that $\mathrm{MB}$ involvement does not directly correspond to HIE severity. Furthermore, in some patients, the $\mathrm{MB}$ were the only structure that showed a signal change, again suggesting that this region has a different sensitivity to hypoxia than other brain structures. The duration and severity of the hypoxic-ischemic episode may be key determinants of the MBs sensitivity. For example, a short acute moment of hypoperfusion of the brain due to HIE-triggered cardiac malfunctioning may be sufficient to cause damage to the MBs. This could explain those patients with abnormal MB signal but otherwise normal MR imaging. The lack of MB pathology in some severely asphyxiated patients would, however, suggest some degree of neuroprotection in these patients, which needs to be investigated further.

Given the importance of the MBs for episodic memory, ${ }^{32}$ it is likely that $\mathrm{MB}$ pathology in patients with HIE contributes to memory impairment that can be associated with this patient group. However, the concomitant hippocampal damage makes it difficult to attribute specific impairment to the MBs. In the current study, there were 13 patients (42\%) in whom the initial scan showed MB signal rise without any visible signal changes of other structures within the Papez circuit. These patients would be of particular interest in terms of neuropsychological follow-up. While there are reports of localized $\mathrm{MB}$ infarcts causing amnesia in adults, ${ }^{33,34}$ it is not known whether developmentally acquired MB damage would produce similar neuropsychological outcomes.

In 1 case, we noted an acute T2-weighted signal change in the MBs, but there was no observable atrophy on follow-up MR imaging. Unfortunately, long-term clinical follow-up was not available in this case. It is possible that the MBs were dysfunctional despite no obvious cell loss; alternatively, it is possible that there was some degree of neuroprotection against subsequent cell loss. Again, this case highlights the importance of assessing individual differences in response to HIE because this could prove critical in generating treatment that provides additional neuroprotection to those populations most at risk.

While $13 \%$ of examined cases were found to have signal change in the MBs, this is likely to be an underestimation of total occurrence. Given the size of the MBs, particularly in neonates, a 4-mm section thickness results in the MBs being poorly visualized. ${ }^{3}$ Furthermore, the location of the MBs at the base of the brain can produce artifacts, which obscure imaging details. This problem was exacerbated further with the ADC maps, but in some patients, we noted low ADC signal at the MBs suggesting ischemia. This emphasizes the need to focus on the T2 sequence because in all 6 patients in whom the DWI was positive, the T2 imaging findings were also positive, but the reverse was not found to be true.

In summary, this study has shown that abnormal MB signal can be found in the acute period following HIE in neonates, and this abnormal signal appears an effective predictor of subsequent MB atrophy. The sensitivity for damage to the MBs does not seem to be directly related to the severity of hypoxia, as indicated by standard measures, and does not appear to be affected by treatment involving whole-body cooling. Given the importance of the MBs for memory, this further highlights the need to more closely examine this structure in patient groups with both HIE and other conditions associated with reduced brain oxygen levels.

\section{Conclusions}

This study highlights the need to carefully assess the MBs in neonates with HIE. For the most accurate assessment of the MBs, high-resolution images are needed using the thinnest slices possible ( $\leq 2 \mathrm{~mm}$ for the T2-weighted sequence). Furthermore, the use of a dedicated thin-section DWI sequence, adjusted for the skull base, would improve the accuracy of identifying MB involvement in this patient group. Repeated scanning for a range of days after birth could also be informative for identifying the time window most sensitive to MB signal change. With detailed clinical assessment and subsequent MR imaging and neurocognitive follow-up at school age, it may be possible to determine the consequences of early-onset MB pathology and identify those patients most at risk during the acute phase.

Disclosures: Seralynne D. Vann—RELATED: Grant: Wellcome Trust, Comments: My salary is supported by the Wellcome Trust by way of a Senior Research Fellowship*; UNRELATED: Grants/Grants Pending: Wellcome Trust, Comments: I am supported by a Wellcome Trust Fellowship. * *oney paid to the institution.

\section{REFERENCES}

1. Vann SD. Re-evaluating the role of the mammillary bodies in memory. Neuropsychologia 2010;48:2316-27 CrossRef Medline

2. Sziklas V, Petrides M. Memory and the region of the mammillary bodies. Prog Neurobiol 1998;54:55-70 CrossRef Medline

3. Vann SD, Aggleton JP. The mammillary bodies: two memory systems in one? Nat Rev Neurosci 2004;5:35-44 CrossRef Medline

4. Kopelman MD. The Korsakoff syndrome. Br J Psychiatry 1995; 166:154-73 CrossRef Medline

5. Tsivilis D, Vann SD, Denby C, et al. A disproportionate role for the fornix and mammillary bodies in recall versus recognition memory. Nat Neurosci 2008;11:834-42 CrossRef Medline

6. Kumar R, Birrer BVX, Macey PM, et al. Reduced mammillary body volume in patients with obstructive sleep apnea. Neurosci Lett 2008;438:330-34 CrossRef Medline

7. Bernstein HG, Krause S, Krell D, et al. Strongly reduced number of parvalbumin-immunoreactive projection neurons in the mammillary bodies in schizophrenia: further evidence for limbic neuropathology. Ann N Y Acad Sci 2007;1096:120-27 CrossRef Medline

8. de Vries LS, Groenendaal F. Patterns of neonatal hypoxic-ischaemic brain injury. Neuroradiology 2010;52:555-66 CrossRef Medline

9. Alderliesten T, Nikkels PG, Benders MJ, et al. Antemortem cranial MRI compared with postmortem histopathologic examination of the brain in term infants with neonatal encephalopathy following perinatal asphyxia. Arch Dis Child Fetal Neonatal Ed 2013;98:F30409 CrossRef Medline

10. Kasdorf E, Engel M, Heier L, et al. Therapeutic hypothermia in neonates and selective hippocampal injury on diffusion-weighted magnetic resonance imaging. Pediatr Neurol 2014;51:104-08 CrossRef Medline

11. Maneru C, Serra-Grabulosa JM, Junque C, et al. Residual hippocampal atrophy in asphyxiated term neonates. J Neuroimaging 2003; 13:68-74 CrossRef Medline

12. Gadian DG, Aicardi J, Watkins KE, et al. Developmental amnesia associated with early hypoxic-ischaemic injury. Brain 2000;123(Pt 3):499-507 CrossRef Medline 
13. Cooper JM, Gadian DG, Jentschke S, et al. Neonatal hypoxia, hippocampal atrophy, and memory impairment: evidence of a causal sequence. Cereb Cortex 2015;25:1469-76 CrossRef Medline

14. Dzieciol AM, Bachevalier J, Saleem KS, et al. Hippocampal and diencephalic pathology in developmental amnesia. Cortex 2017;86:3344 CrossRef Medline

15. Loftus M, Knight RT, Amaral DG. An analysis of atrophy in the medial mammillary nucleus following hippocampal and fornix lesions in humans and nonhuman primates. Exp Neurol 2000; 163:180-190 CrossRef Medline

16. Johkura K, Naito M. Wernicke's encephalopathy-like lesions in global cerebral hypoxia. J Clin Neurosci 2008;15:318-319 CrossRef Medline

17. Schmidtke K. Wernicke-Korsakoff syndrome following attempted hanging. Rev Neurol (Paris) 1993;149:213-16 Medline

18. Cowan F, Rutherford M, Groenendaal F, et al. Origin and timing of brain lesions in term infants with neonatal encephalopathy. Lancet 2003;361:736-42 CrossRef Medline

19. Alderliesten T, de Vries LS, Benders MJ, et al. MR imaging and outcome of term neonates with perinatal asphyxia: value of diffusionweighted MR imaging and ${ }^{\mathbf{1}} \mathbf{H}$ MR spectroscopy. Radiology 2011; 261:235-42 CrossRef Medline

20. Alderliesten T, de Vries LS, Staats L, et al. MRI and spectroscopy in (near) term neonates with perinatal asphyxia and therapeutic hypothermia. Arch Dis Child Fetal Neonatal Ed 2017;102:F147-52 CrossRef Medline

21. Alderliesten T, de Vries LS, Khalil Y, et al. Therapeutic hypothermia modifies perinatal asphyxia-induced changes of the corpus callosum and outcome in neonates. PLoS One 2015;10:e123230 CrossRef

22. Weeke LC, Groenendaal F, Mudigonda K, et al. A novel magnetic resonance imaging score predicts neurodevelopmental outcome after perinatal asphyxia and therapeutic hypothermia. J Pediatrics 2018;192:33-40.e2 CrossRef Medline

23. Bednarek N, Mathur A, Inder T, et al. Impact of therapeutic hypothermia on MRI diffusion changes in neonatal encephalopathy. Neurology 2012;78:1420-27 CrossRef Medline
24. Papez JW. A proposed mechanism of emotion. Archives of Neurology and Psychiatry 1937;38:725-43 CrossRef

25. Vann SD, Nelson AJ. The mammillary bodies and memory: more than a hippocampal relay. Prog Brain Res 2015;219:163-85 CrossRef Medline

26. Froudist-Walsh S, Browning PGF, Croxson PL, et al. The rhesus monkey hippocampus critically contributes to scene memory retrieval, but not new learning. J Neurosci 2018;38:7800-08 CrossRef Medline

27. Vortmeyer AO, Hagel C, Laas R. Hypoxia-ischemia and thiamine deficiency. Clin Neuropathol 1993;12:184-90 Medline

28. Kumar R, Woo MA, Birrer BVX, et al. Mammillary bodies and fornix fibers are injured in heart failure. Neurobiol Dis 2009;33:236-42 CrossRef Medline

29. Beh SC, Frohman TC, Frohman EM. Isolated mammillary body involvement on MRI in Wernicke's encephalopathy. J Neurol Sci 2013;334:172-75 CrossRef Medline

30. Kornreich L, Bron-Harlev E, Hoffmann C, et al. Thiamine deficiency in infants: MR findings in the brain. AJNR Am J Neuroradiol 2005;26:1668-74 Medline

31. Zuccoli G, Siddiqui N, Bailey A, et al. Neuroimaging findings in pediatric Wernicke encephalopathy: a review. Neuroradiology 2010; 52:523-29 CrossRef Medline

32. Dillingham CM, Milczarek MM, Perry JC, et al. Mammillothalamic disconnection alters hippocampo-cortical oscillatory activity and microstructure: implications for diencephalic amnesia. J Neurosci 2019;39:6696-6713 CrossRef Medline

33. Amuluru K, Filippi CG, Lignelli A. Acute amnesia due to isolated mammillary body infarct. J Stroke Cerebrovasc Dis 2015;24:e303-05 CrossRef Medline

34. Male S, Zand R. Isolated mammillary body infarct causing global amnesia: a case report. J Stroke Cerebrovasc Dis 2017;26:e50-52 CrossRef Medline

35. Denby CE, Vann SD, Tsivilis D, et al. The frequency and extent of mammillary body atrophy associated with surgical removal of a colloid cyst. AJNR Am J Neuroradiol 2009;30:736-43 CrossRef Medline 